Delft University of Technology

\title{
Time Delay Estimation Based on Multi-band Multi-carrier Signal in Multipath Environments
}

Dun, Han; Tiberius, Christiaan; Janssen, Gerard; Diouf, Cherif

DOI

10.33012/2019.16958

Publication date

2019

Document Version

Accepted author manuscript

Published in

Proceedings of the 32nd International Technical Meeting of the Satellite Division of The Institute of

Navigation (ION GNSS+ 2019)

\section{Citation (APA)}

Dun, H., Tiberius, C., Janssen, G., \& Diouf, C. (2019). Time Delay Estimation Based on Multi-band Multicarrier Signal in Multipath Environments. In Proceedings of the 32nd International Technical Meeting of the Satellite Division of The Institute of Navigation (ION GNSS+ 2019): September 16 - 20, 2019 Hyatt Regency Miami Miami, Florida (pp. 2299-2313). ION - Inst. of Navigation. https://doi.org/10.33012/2019.16958

\section{Important note}

To cite this publication, please use the final published version (if applicable).

Please check the document version above.

\section{Copyright}

Other than for strictly personal use, it is not permitted to download, forward or distribute the text or part of it, without the consent of the author(s) and/or copyright holder(s), unless the work is under an open content license such as Creative Commons.

Takedown policy

Please contact us and provide details if you believe this document breaches copyrights. We will remove access to the work immediately and investigate your claim. 


\title{
Time Delay Estimation Based on Multi-band Multi-carrier Signal in Multipath Environments
}

\author{
Han Dun ${ }^{1}$, Christian C. J. M. Tiberius ${ }^{1}$, Gerard J. M. Janssen ${ }^{2}$, and Cherif Diouf ${ }^{1}$ \\ ${ }^{1}$ Geoscience and Remote Sensing, Delft University of Technology, Delft, The Netherlands \\ ${ }^{2}$ Circuits and Systems, Delft University of Technology, Delft, The Netherlands
}

\begin{abstract}
BIOGRAPHIES
Han Dun received his BSc degree in Communication Engineering and MSc degree in Communication and Information Engineering from Shanghai University, China, in 2013 and 2016, respectively. From 2013 to 2016, he also worked at the key laboratory of specialty fiber optics and optical access network in Shanghai University, where he contributed to the real-time optical OFDM-PON. He is currently pursuing his PhD degree in the department of Geoscience and Remote Sensing, Delft University of Technology. His research interests include digital communication theory, wireless localization, and statistical signal processing.
\end{abstract}

Dr. Christian C.J.M. Tiberius received his PhD degree in 1998 from the Delft University of Technology, Delft, The Netherlands, on recursive data processing for kinematic GPS surveying. He is currently an Associate Professor with the Geoscience and Remote Sensing (GRS) department, Delft University of Technology. His research interest lies in navigation, with GNSS and terrestrial radio positioning. He is, and has been responsible, for more than 15 years, for many projects in the area of navigation with national and international agencies, like ESA, and industry, as well.

Dr. Gerard J. M. Janssen received the M.Sc. E.E. degree from Eindhoven University of Technology, Eindhoven, The Netherlands, in 1986 and the Ph.D. degree from Delft University of Technology, Delft, The Netherlands, in 1998. He is currently an Associate Professor with the Circuits and Systems Group, Delft University of Technology. His research interests include wireless communication, particularly narrowband multiuser detection, digital modulation techniques, channel modeling, diversity techniques, and ultra wideband communications and positioning.

Dr. Cherif Diouf received a PhD degree in non-linear system modeling applied to VLSI electronic circuits in 2014 from the Université de Bretagne Occidentale (France). He then worked at the Ecole Nationale d'Ingénieurs of Brest on DSP techniques to optimize the performance of optical OFDM communication systems. In 2015, he joined the French Oceanographic institute (Ifremer). As a postdoctoral researcher, he worked on the development of a power-over-fiber prototype to allow extensions of a sea-bed observatory. Recently, he was working as an embedded systems engineer on autonomous floats. In 2018, he joined the SuperGPS research team as a postdoctoral researcher. He is interested in blackbox modeling and DSP techniques, HW/SW implementation and electro-optical systems.

\begin{abstract}
The matched filter is the most common approach for time delay estimation and ranging in positioning systems. The accuracy is mainly determined by the signal bandwidth and multipath propagation condition. Instead of occupying an enormous signal bandwidth, aggregating multiple signal bands, which are transmitted either simultaneously or sequentially from the same transmitter, can still provide a very high time resolution due to its large virtual signal bandwidth. This paper discusses time delay estimation based on multiband signals, considering precision, range ambiguity and resistance to multipath. Combining carrier phases from different bands, which are physically not perturbed by a sampling frequency offset, can also mitigate the bias of time delay estimation due to the sampling frequency error. Simulation results show that using two groups of multiband signals, which are sparsely placed in the signal spectrum, can significantly improve the accuracy of time delay estimation in the presence of multipath and sampling frequency offset.
\end{abstract}

\section{INTRODUCTION}

In a ranging system for positioning and navigation, the propagation time delay (i.e., path delay or time of flight) can be estimated based on a matched filter [1], which can be interpreted as a maximum likelihood method considering additive white Gaussian noise (AWGN). Hence, time delay estimation is to maximize the correlation function between the received signal and the locally 
generated reference signal. The accuracy of time delay estimation is inversely proportional to the signal bandwidth. Thus, a large signal bandwidth is required for precise ranging and positioning, based on time delay estimation only. However, for those signals with a large signal bandwidth (e.g., an ultra-wide band (UWB) signal [2]), the receiver requires an ADC with a very high sampling rate and strong processing power. Alternatively, we explore the idea of combining multiple signal bands to increase the virtual signal bandwidth.

For a multiband system, the channel estimation based on a known transmitted signal, such as orthogonal frequency division multiplexing (OFDM) training symbols, is carried out to retrieve the channel impulse response. Xu et al. [3] average the channel estimates from different signal bands to obtain better estimation results. Wolf et al. [4] proposed a multi-channel ranging protocol for low power wide area network (LPWAN) by aggregating sequentially transmitted narrowband signals. The propagation delay is retrieved from the estimated channel impulse response after ordinary inverse Fourier transform. Vasisht et al. [5] proposed a method for stitching time measurements across WiFi frequency bands which have non-uniform spacing. Based on the non-uniform Fourier transform of channel measurements from different bands, the system can retrieve the multipath profile and determine the propagation delay of the LoS path. All these methods require the (inverse) Fourier transform to recover the channel impulse response.

Instead of recovering a full channel impulse response, we can simply apply the matched filter in the time domain to determine the propagation time delay. Recently, Yang and Soloviev [6] proposed a concept of variable IF (intermediate frequency) correlation (VIC) for a GPS receiver. The GPS signal is down-converted to different IFs. Then, the correlation functions from different IFs are pinched to emulate a relatively large virtual signal bandwidth. Consequently, time delay estimation can be improved in multipath condition. In this paper, we also restrict ourselves solely to use the simple matched filter method to estimate the propagation delay, and discuss the relation between the accuracy of time delay estimation and the signal format.

In the presence of AWGN, the precision of time delay estimation can be assessed by the Cramér-Rao lower bound (CRLB) [7]. It provides insight into how to design a signal for the purpose of ranging and positioning. In order to attain a better ranging precision, we should place more power at the edges of the available signal spectrum [8] (similar to the concept of a BOC signal [9]). In an extreme case, we can simply transmit two sinusoids with maximum frequency separation. However, the correlation peak, from which the propagation delay can be retrieved, occurs periodically in the cross correlation function. The period of the correlation function relies on the frequency difference of the two sinusoids (i.e., beat frequency). Consequently, based on the correlation function that contains multiple peaks with identical amplitude, time delay estimation becomes ambiguous. There is a trade-off between precision and ambiguity. In fact, the CRLB only considers the precision of time delay estimation, not the ambiguity.

On the other hand, the ambiguity problem, and the presence of side lobes when more than two sinusoids (e.g., OFDM subcarriers) are used, also makes time delay estimation in such a system less robust to multipath channels (i.e., easily be affected by multipath). The cross correlation function of the received signal is a sum of the correlation function of the received LoS signal and those of the reflections. When the cross correlation function of the signal itself contains multiple peaks, then the correlation function of the received signal in a multipath channel will be largely affected by reflections that have relatively strong power.

In this paper, we investigate signal design for the purpose of ranging and positioning, considering not only precision of time delay estimation, but also ambiguity of time delay estimation, and robustness in a multipath channel. In addition, we consider an OFDM signal as a basic signal format in this paper, because it provides us the flexibility to explore the signal spectrum, and has been also used in LTE [10] and IEEE802.11p (WiFi-p) [11], and can be also adapted for a terrestrial radio positioning system.

The paper is organized as follows. A brief introduction of maximum likelihood based time delay estimation is given in section II. Time estimation based on a single band, multiband, and sparse multiband signals are explained in section III, IV and V, respectively. The impact of the sampling frequency offset on time delay estimation is analyzed in section section VI. Section VII presents simulation results of time delay estimation in the presence of a sampling frequency offset and multipath. At the end of this paper, we formulate practical guidelines for the design of a multiband signal for time delay estimation and ranging.

\section{MAXIMUM LIKELIHOOD TIME DELAY ESTIMATION}

The propagation time delay, as the pseudo-range code observation in GNSS, is a basic measurement for ranging and positioning. It can be easily estimated based on a matched filter method, which can be also interpreted as a maximum likelihood (ML) method considering Gaussian noise. Hence, time delay estimation is to maximize the correlation function between the received signal $r[n]$ and the locally generated reference signal $s[n]$. For ease of notation, the propagation delay $\tilde{\tau}$ is normalized by the sampling 
interval $T_{s}$. Afterwards, the correlation function is defined by

$$
R_{r s}(\tilde{\tau})=\frac{1}{N} \sum_{n=0}^{N-1} r[n] s^{*}[n-\tilde{\tau}], \quad \tilde{\tau} \in \mathbb{R},
$$

where $n$ denotes the sample index, $(\cdot)^{*}$ denotes the conjugate operation. Afterwards, the propagation time delay is estimated by

$$
\hat{\tau}=\underset{\tilde{\tau}}{\arg \max }\left\{\left|R_{r s}(\tilde{\tau})\right|\right\}
$$

To evaluate the performance of time delay estimation, the CRLB determines a lower bound on the variance of any unbiased estimator [7]. Without considering any multipath, offsets and interference, the CRLB of time delay estimation is given as follows

$$
\operatorname{var}\left(\hat{\tau} T_{S}\right) \geq \frac{1}{\mathrm{SNR}_{\bar{F}}^{2}}
$$

where $\bar{F}^{2}$ is the mean square bandwidth of the signal (i.e., Gabor bandwidth) [7], and is defined by

$$
\bar{F}^{2}=\frac{\int_{-\infty}^{\infty}(2 \pi F)^{2}|S(F)|^{2} d F}{\int_{-\infty}^{\infty}|S(F)|^{2} d F}
$$

and $S(F)$ is the Fourier transform of the reference signal $s[n]$.

Based on (3) and (4), increasing the signal-to-noise ratio (SNR) as well as increasing the signal bandwidth can result in a better accuracy of time delay estimation. On the other hand, when the SNR and the signal bandwidth are fixed, the accuracy can still be further improved by maximizing $\bar{F}^{2}$ in (4). This provides us some insights into signal design for ranging and positioning, and it will be further discussed in the following sections.

\section{TIME DELAY ESTIMATION BASED ON SINGLE BAND OFDM SIGNAL}

Considering an OFDM signal as a generic multi-carrier signal waveform for time delay estimation and ranging, the transmitted baseband OFDM signal with $N$ subcarriers is given by [12]

$$
s[n]=\frac{1}{\sqrt{N}} \sum_{i=-N / 2}^{N / 2-1} c_{i} \exp \left(j 2 \pi \frac{i}{N} n\right),
$$

where $c_{i}$ is the data modulated on the $i$-th pilot subcarrier. After the DAC, the continuous OFDM signal will be modulated on a central carrier with frequency $f_{c}$. Thus, the pass-band signal is given by

$$
s_{p}(t)=\mathfrak{R}\left\{s(t) \exp \left(j 2 \pi f_{c} t\right)\right\} ; \quad t=n T_{s}, \quad T_{s}=1 / B,
$$

where $\mathfrak{R}\{\cdot\}$ denotes the real part of a complex value, $T_{s}$ denotes the sampling interval, which is the inverse of the signal bandwidth $B$.

In order to let time delay estimation be unbiased, we first consider a simple single-path channel. So that the accuracy can be evaluated by the CRLB, which also gives us more insight into how the signal waveform is linked to the performance of time delay estimation. A simple single path channel is given by the following channel impulse response (CIR),

$$
h(t, \tau)=\alpha \delta\left(t-\tau T_{s}\right), \quad\{\alpha, \tau\} \in \mathbb{R}^{+},
$$

in which $\alpha$ denotes the attenuation due to the propagation, typically with $\alpha \leq 1$. Generally, we normalize the attenuation of the LoS path to one. In (7), $\tau$ denotes the propagation delay normalized by the sample interval $T_{s}$.

Here, we assume that the system can run stably in a certain time interval, so that the initial phase offset and the constant carrier frequency offset of the receiver with respect to the transmitter can be compensated by an offline calibration procedure. In addition, for the ease of notation, we do not yet consider the sampling frequency offset, but its impact on time delay estimation will be analyzed later in section VI. Therefore, after the down-converter and ADC, the received baseband signal can be written as

$$
r[n]=\frac{1}{\sqrt{N}} \sum_{i=-N / 2}^{N / 2-1} c_{i} \exp \left(j 2 \pi \frac{i}{N} n\right) \exp \left(-j 2 \pi\left(i \Delta f+f_{c}\right) \tau T_{s}\right), \quad \Delta f=B / N=1 / N T_{s},
$$

where $\Delta f$ denotes the subcarrier spacing. 


\section{a. CRLB of time delay estimation based on OFDM signal}

Since with (7) we do not consider any interference and offset, time delay estimation is unbiased. First, the CRLB of time delay estimation based on OFDM signals is derived in [8,13,14]. According to (3), given a fixed signal bandwidth (i.e., sampling interval), the CRLB is given by

$$
\operatorname{CRLB}(\tau)=\frac{1}{8 \pi^{2} \operatorname{SNR} \frac{\sum_{i=-N / 2}^{N / 2-1} i^{2} \Delta f^{2} p_{i}^{2}}{\frac{1}{N} N}}=\frac{1}{8 \pi^{2} \operatorname{SNR} \Delta f^{2} \sum_{i \in N_{a}} p_{i}^{2} i^{2}}
$$

where $N_{a}$ is the subset of activated sub-carriers for ranging and positioning, $p_{i}$ denotes the allocated power to the $i$-th subcarrier. Thus, for ML-based time delay estimation, the optimal power distribution of OFDM signal is allocating more power at the edges of the spectrum due to the term $\sum p_{i}^{2} i^{2}$. For an extreme case, we should transmit only two edge subcarriers and mute the other subcarriers [8] for ranging and positioning, so all signal power can be placed really at both edges of the available spectrum.

\section{b. correlation function}

We can easily derive a theoretical lower bound of time delay estimation based on the CRLB, but in practice, the propagation delay is obtained or estimated from the correlation function as shown in (1). Therefore, we will discuss some properties of the correlation function and link it back to the CRLB derived in the previous subsection.

In order to derive a closed-form expression of the cross correlation function, we start with a basic OFDM signal but only transmit pilots, its subcarrier spacing and the power of pilots are assumed to be the same. The baseband OFDM signal in discrete time is given by

$$
s[n]=\frac{1}{\sqrt{M}} \sum_{i=-M / 2}^{M / 2-1} c_{i} \exp \left(j 2 \pi \frac{i P}{N} n\right), \quad n=0,1, \ldots, N-1, \quad M=\left\lfloor\frac{N-1}{P}\right\rfloor+1,
$$

in which $M$ denotes the number of pilots out of the total number of subcarriers $N$ for ranging, $P$ denotes the interval between two adjacent pilots, $\lfloor\cdot\rfloor$ denotes rounding down. For simplicity, we consider a BPSK modulation on every subcarrier (i.e., $\left.\left|c_{i}\right|=1\right)$. Then, the cross-correlation function is derived as follows

$$
R_{r s}(\tilde{\tau})=\frac{\exp \left(-j 2 \pi f_{c} \tau T_{s}\right)}{M} \exp \left(j 2 \pi \frac{M P}{2 N}(\tau-\tilde{\tau})\right) \exp \left(-j 2 \pi \frac{P}{N} \frac{M-1}{2}(\tau-\tilde{\tau})\right) \frac{\sin \left(2 \pi \frac{P}{N} \frac{M}{2}(\tau-\tilde{\tau})\right)}{\sin \left(2 \pi \frac{P}{N} \frac{1}{2}(\tau-\tilde{\tau})\right)} .
$$

The derivation can be found in Appendix A. Therefore, the correlation function $R_{r s}(\hat{\tau})$ will reach its the maximum, when the time delay estimate $\hat{\tau}$ is aligned with the propagation delay $\tau$. Since ML-based time delay estimation can rely only on the amplitude of the cross-correlation function, the modulus of the correlation function derived in (11) is given by

$$
C_{r s}(\tilde{\tau})=\left|R_{r s}(\tilde{\tau})\right|=\left|\frac{1}{M} \frac{\sin \left(2 \pi \frac{P}{N} \frac{M}{2}(\tau-\tilde{\tau})\right)}{\sin \left(2 \pi \frac{P}{N} \frac{1}{2}(\tau-\tilde{\tau})\right)}\right|,
$$

in which $\tilde{\tau}$ is again normalized by the sampling interval $T_{s}$. This correlation function, as a function of $\tilde{\tau}$ as shown in Fig. 1, potentially contains multiple main lobes, and also side lobes when more than two subcarriers are used for time delay estimation.

\section{Precision}

We can further analyze the characteristics of the amplitude of the cross correlation shown in (12). Let $t_{0,1}$ denote the first zero point of the cross correlation function, which also represents the width of the main lobe in the cross correlation function,

$$
t_{0,1}=\frac{N}{P M}\left(T_{s}\right)
$$

In terms of time delay estimation and ranging, the narrower the main lobe is, the more precise time delay estimation can be. For example, when time delay estimation is based on the early-minus-late (EML) tracking loop, the variance of delay estimation depends on the slope of the discriminator function [15]. When the value of the peak in the correlation function is fixed (i.e., the signal power is fixed), a small first zero point can result in a steep slope. A peaked main lobe in the correlation function leads to a better precision of time delay estimation. Therefore, given an available signal bandwidth, in order to improve the precision of time delay estimation, we should insert pilots as few as we can, and mute other subcarriers, so that the pilot spacing $P$ is as large as possible, and more power can be placed at the edges of the signal spectrum. This is also in line with the conclusion derived from the CRLB in (9). 

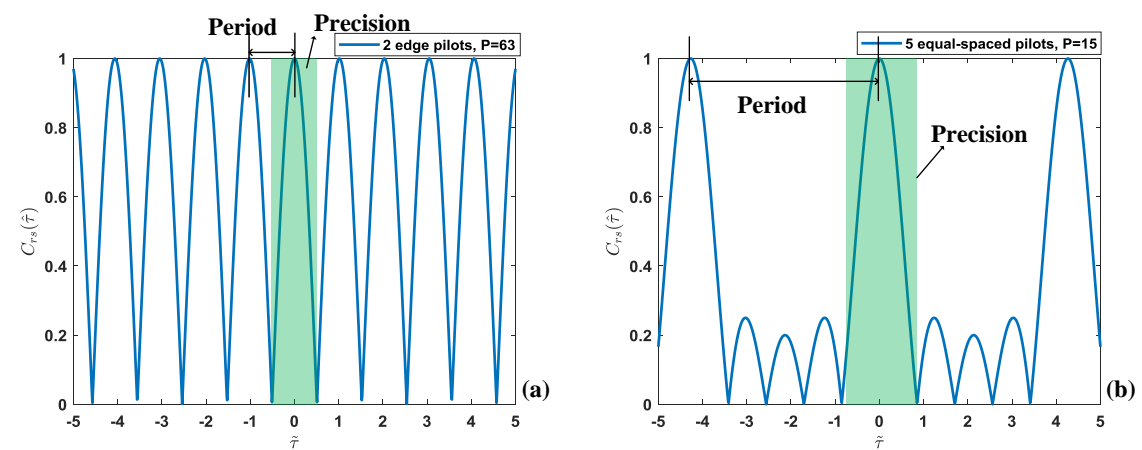

Figure 1: The amplitude of the cross-correlation function of a two edge pilots (a), and a five equally spaced pilots (b). Besides the pilot subcarriers, other subcarriers are muted. The overall signal power is identical in both cases and the signal bandwidth is assumed to be $10 \mathrm{MHz}$. The horizontal axis shows the time delay estimate normalized by the sample interval (i.e., $T_{s}=10^{-7} \mathrm{~s}$ ). Since the actual propagation delay is offset to zero for this graph, the main lobe of the correlation function occurs at $\tilde{\tau}=0$.

\section{Ambiguity}

In addition, the main lobe periodically appears in the cross correlation function. Its period $T_{0}$ can be derived from the denominator in (12) and is given by

$$
T_{0}=\frac{N}{P}\left(T_{s}\right) .
$$

Given a fixed signal bandwidth, the more pilots we insert (i.e., smaller $P$ ), the larger the period of the correlation function becomes. Its period also determines the unambiguous ranging distance.

For example, given a fixed signal bandwidth of $10 \mathrm{MHz}$, if we use only two edge pilot subcarriers (i.e., the $\{-32,31\}$-th subcarriers, when $N=64, M=2, P=63$ ), the main lobe will occur almost every $T_{s}$. The cross correlation function is shown in Fig. 1(a). In such a case, the unambiguous ranging distance is about $30 \mathrm{~m}$. We can insert more pilots to increase the period of the correlation function. If we insert $M=5$ pilots with an equal spacing (i.e., the $\{-32,-17,-2,13,28\}$-th subcarrier, $P=15$ ), based on (14) the period of the correlation function will be $4.27 T_{s}$, and the correlation function is presented in Fig. 1(b). The unambiguous ranging distance is extended to about $128 \mathrm{~m}$. Inserting more pilots reduces the ambiguity of time delay estimation, but it also sacrifices precision, because the main lobe becomes wider.

Note that in practice, in the presence of noise, a large side lobe may get bigger than the actual main lobe, which can also lead to an ambiguity problem (also referred to as false or incorrect detection). In addition, if we consider the data $c_{i}$ modulated on pilot subcarriers, then there is no ambiguity introduced to time delay estimation beyond $N T_{s}$ (or $\left(N_{g}+N\right) T_{s}$, considering a cyclic prefix with $N_{g}$ samples). However, ambiguity can still exist within $N T_{s}\left(\right.$ or $\left.\left(N_{g}+N\right) T_{s}\right)$.

\section{Multipath resistance}

Considering a multipath channel with the following CIR,

$$
h(t, \tau)=\sum_{p=1}^{D} \alpha_{p} \delta\left(t-\tau_{p} T_{s}\right), \quad \tau=\left[\begin{array}{llll}
\tau_{1} & \tau_{2} & \ldots & \tau_{D}
\end{array}\right]
$$

the cross correlation function of the received signal will be the sum of the correlation functions of the signals from all $D$ paths (i.e., LoS and reflections). The correlation function of a received multipath signal is an attenuated correlation function of the LoS signal and shifted by the relative delay. Hence, time delay estimation will be affected by the side lobes and the repeated main lobes through the ambiguity. Given a fixed $10 \mathrm{MHz}$ signal bandwidth, based on only two edge subcarriers, time delay estimation can be easily affected by a reflection in a multipath channel. However, ranging based on five equally spaced pilots (i.e., the $\{-32,-17,-2,13,28\}$-th subcarrier) can be more robust in multipath conditions, because the amplitude of the side lobes is significantly reduced as shown in Fig. 1(b). In addition, Fig. 2 presents the envelope of time delay estimation error in a two-path multipath channel. Compared with the case that only two edge subcarriers are used for time delay estimation, the impact of a reflection with a relative delay from $1 T_{s}$ to $3 T_{s}(30-90 \mathrm{~m}$ in distance) can be largely mitigated, when five equally spaced pilots are used. 


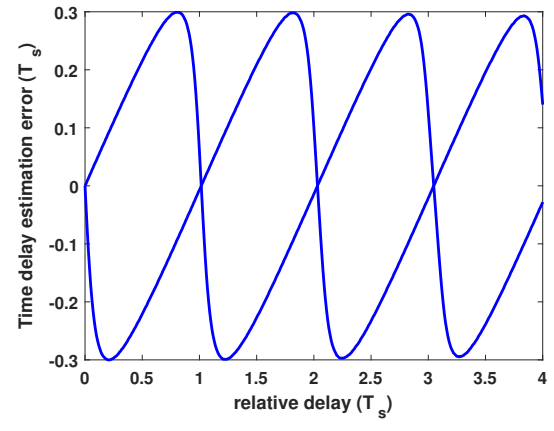

(a)

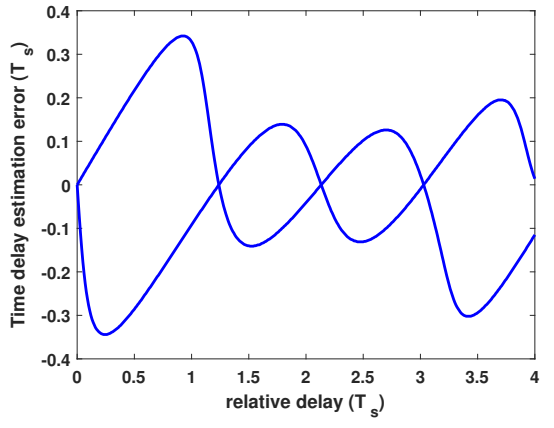

(b)

Figure 2: The envelope of the multipath time delay estimation error, as a single band OFDM signal with only $M=2$ edge subcarriers (a) and $M=5$ equally spaced pilots are transmitted for time delay estimation and ranging, and the relative attenuation $\alpha_{2}=0.8$.

Given a fixed signal bandwidth, we should let the pilot spacing be as large as possible, in order to obtain a better precision. It also means that less subcarriers should be used for time delay estimation and ranging. But even so, in practice, the ambiguity and the robustness of time delay estimation in a multipath environment should be taken into account. In accordance to the required unambiguous distance and the typical channel characteristic, we can compute the required period of the correlation function, and further determine the pilot spacing and the minimum number of pilots for the purpose of time delay estimation and ranging.

\section{TIME DELAY ESTIMATION BASED ON MULTIBAND OFDM SIGNAL}

In a single band signal, the precision of time delay estimation and the resistance to multipath are limited by the signal bandwidth. On the one hand, we can simply transmit a wideband signal to obtain a high time resolution and a better ranging accuracy. However, it requires a lot of spectrum resources, which in practice is not available. On the other hand, we can aggregate multiple signal bands as shown in Fig. 3, which can be transmitted and received either simultaneously or sequentially, to create a large virtual signal bandwidth. The signal could be received at the same time in parallel via multiple RF front-ends which are dedicated to different bands, or the signal is received by a single RF front-end, in which the central carrier is tuned into different frequencies at different time slots to acquire the signal from different bands. Similarly, in order to obtain a closed-form expression of the cross correlation function based on a multiband signal, we assume that the central carrier spacing $\Delta f_{c}$ and modulation are the same across all adjacent bands, but it can be different.

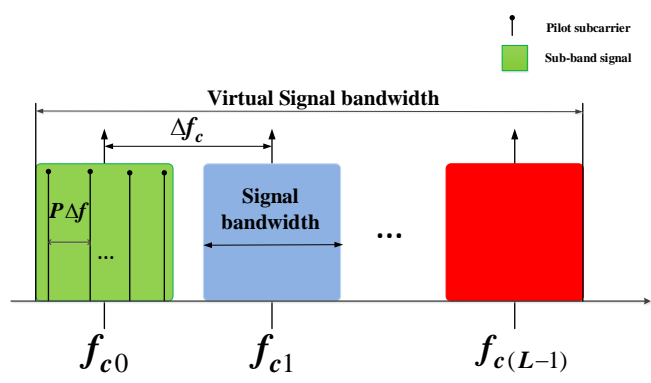

Figure 3: Spectrum of a multiband signal for ranging and positioning, in which the carrier spacing $\Delta f_{c}$ between two adjacent bands is assumed to be the same for the ease of the derivation.

After receiving signals from different bands, the correlation function can be initially computed independently for each band. But eventually we use all signals together to emulate a large virtual signal bandwidth. So that the phase rotation due to the central carrier spacing $\Delta f_{c}$ has to be also taken into account for time delay estimation. Therefore, the locally generated reference signal for the $l$-th band should contain an extra phase rotation due to the corresponding central carrier spacing, and is given by

$$
\begin{aligned}
s_{l}[n, \tilde{\tau}] & =s[n-\tilde{\tau}] \exp \left(-j 2 \pi l \Delta f_{c} \tilde{\tau} T_{s}\right), \quad l=0,1, \ldots, L-1 \\
& =\frac{1}{\sqrt{M}} \sum_{k=-M / 2}^{M / 2-1} c_{k} \exp \left(j 2 \pi \frac{k P n}{N}\right) \exp \left(-j 2 \pi \frac{k \tilde{\tau}}{N}\right) \exp \left(-j 2 \pi l \Delta f_{c} \tilde{\tau} T_{s}\right),
\end{aligned}
$$


where $s[n]$ denotes the baseband signal defined in (10). For simplicity and the ease of derivation, we assume here that $s[n]$ is the same for all bands, but again it can be different. Thus, the locally generated reference signal should correspondingly contain the phase rotation due to the central carrier spacing. It means that the carrier frequency offset and the initial carrier phase offset in each band should be corrected precisely. The cross correlation function of the multiband signal can be now derived by the sum of the correlation function of all bands,

$$
R_{r s}(\tilde{\tau})=\frac{1}{L} \sum_{l=0}^{L-1}\left(\frac{1}{N} \sum_{n=0}^{N-1} r_{l}[n] s_{l}^{*}[n, \tilde{\tau}]\right) .
$$

The derivation of (17) can be found in Appendix B. Then, the amplitude of the cross correlation function of the multiband signals is given by

$$
C_{r s}(\tilde{\tau})=|\underbrace{\frac{1}{L} \frac{\sin \left(2 \pi \Delta f_{c} \frac{L}{2}(\tau-\tilde{\tau}) T_{s}\right)}{\sin \left(2 \pi \Delta f_{c} \frac{1}{2}(\tau-\tilde{\tau}) T_{s}\right)}}_{C_{r s, 2}(\tilde{\tau})} \underbrace{\frac{1}{M} \frac{\sin \left(2 \pi \frac{P}{N} \frac{M}{2}(\tau-\tilde{\tau})\right)}{\sin \left(2 \pi \frac{P}{N} \frac{1}{2}(\tau-\tilde{\tau})\right)}}_{C_{r s, 1}(\tilde{\tau})}| .
$$

The second term, $C_{r s, 1}(\tilde{\tau})$, is referred to as the primary component of the correlation function, and identical to (12). Similarly, the central carriers from different bands can be treated as OFDM 'pilot' subcarriers in the virtual signal bandwidth. Thus, the central carriers from different bands (i.e., central carrier spacing) play the role of the secondary part of the correlation function $C_{r s, 2}(\tilde{\tau})$.

\section{Precision and ambiguity}

The cross correlation function now relies not only on the pilot spacing $P$, but also on the central carrier spacing $\Delta f_{c}$. An example of the cross correlation function $C_{r s}(\tilde{\tau})$ with primary and secondary main lobes is shown in Fig. 4. Based on (13), the first zero point of $C_{r s, 1}(\tilde{\tau})$ is defined as the first primary zero point of the cross correlation function $C_{r s}(\tilde{\tau})$. Similarly, the first zero point of the correlation function of the multiband signal $C_{r s, 2}(\tilde{\tau})$ is defined as the first secondary zero point of $C_{r s}(\tilde{\tau})$, which is given by

$$
t_{0,1}^{\prime}=\frac{1}{L \Delta f_{c}}
$$

Since the first secondary zero point is linked to the inverse of the maximum virtual signal bandwidth, we can simply increase the virtual signal bandwidth to further narrow the main lobe of the cross correlation function and improve the precision of time delay estimation and ranging.

In the same way, we define the period of the function $C_{r s, 1}(\tilde{\tau})$ as the primary period of $C_{r s}(\tilde{\tau})$, given by (14). The period of the function $C_{r s, 2}(\tilde{\tau})$ is then defined as the secondary period of $C_{r s}(\tilde{\tau})$, and is given by

$$
T_{0}^{\prime}=\frac{1}{\Delta f_{c}} .
$$

A larger central carrier spacing results in a smaller secondary period of the cross correlation function $C_{r s}(\tilde{\tau})$.

For example, considering a $10 \mathrm{MHz}$ single band OFDM signal, in which the FFT size is assumed to be $N=64$, and $M=5$ pilots (i.e., the $\{-32,-17,-2,13,28\}$-th subcarrier, $P=15$ ) are inserted with an equal spacing and equal power for ranging. In addition, the central carriers spacing $\Delta f_{c}$, as shown in Fig. 3, is assumed to be $10 \mathrm{MHz}$. In order to achieve a virtual signal bandwidth of $100 \mathrm{MHz}$, we can transmit $L=10$ consecutive bands as in Fig. 3. Alternatively, we can occupy only $L=4$ bands (i.e., the band $\{0,3,6,9\}$ ), then the carrier spacing of those two bands becomes $3 \Delta f_{c}=30 \mathrm{MHz}$. In an extreme case, $L=2$ edge bands (i.e., the band $\{0,9\})$ are used for time delay estimation and ranging. The correlation functions are presented in Fig. 4.

A large central carrier spacing leads to a small secondary period of $T_{0}^{\prime}$ of the correlation function $C_{r s, 2}(\tilde{\tau})$, but the amplitude of the secondary main lobe in the product of $C_{r s}(\tilde{\tau})$ still relies on the primary period $T_{0}$ nevertheless. Therefore, the unambiguous ranging distance is determined by the primary period of the correlation function, which relies only on the pilot spacing (i.e., signal format) in the baseband signal if all bands are modulated with the same baseband signal format. The pilot spacing is 781.3 


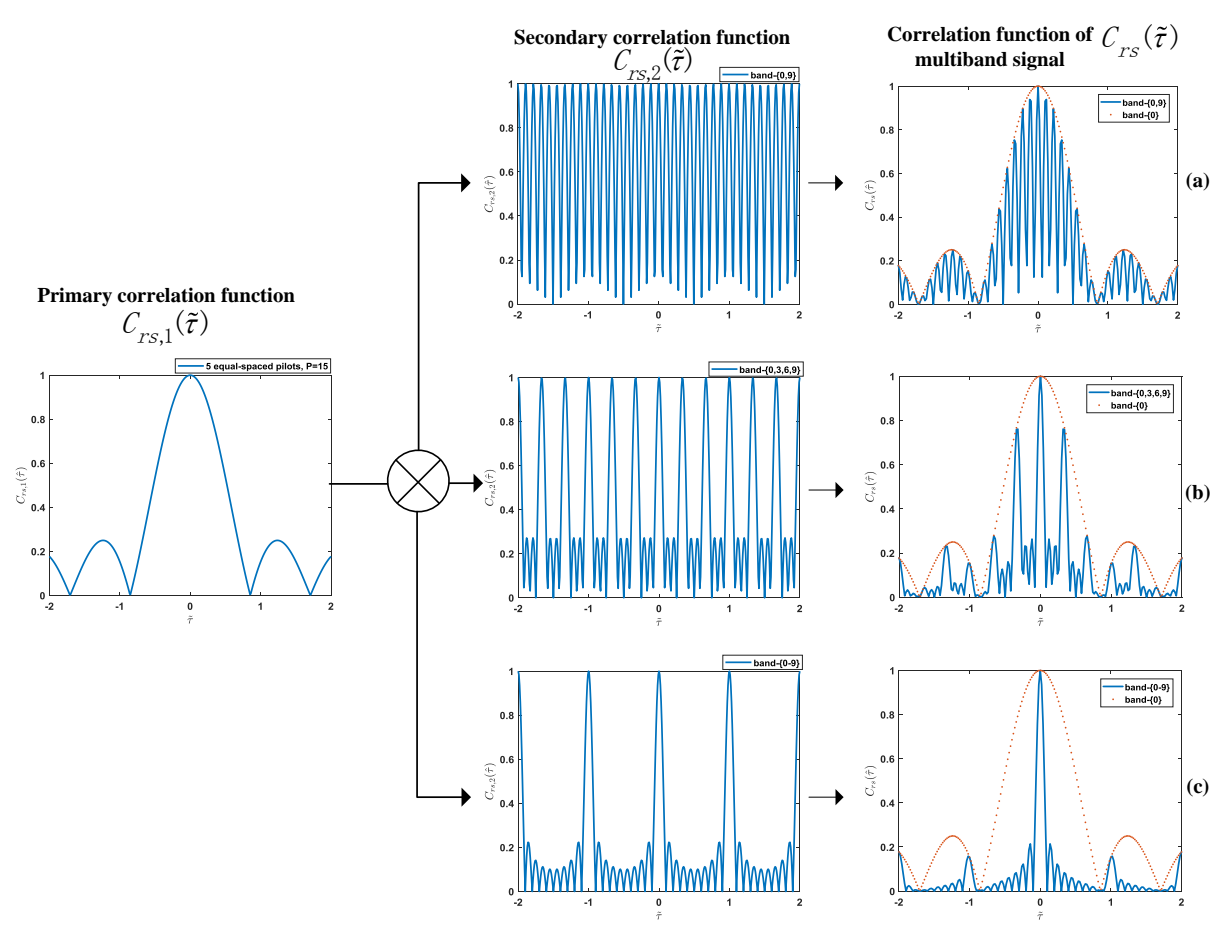

Figure 4: Correlation function of $L=2$ bands (a), $L=4$ bands (b) and $L=10$ bands (c), when the baseband signal bandwidth is $10 \mathrm{MHz}$, and $M=5$ pilots with an equal spacing are inserted in each band for time delay estimation and ranging. The primary correlation function is identical to the one in Fig. 1(b).

$\mathrm{kHz}$ in this example, consequently, based on (14) the unambiguous ranging distance should be about $128 \mathrm{~m}$ no matter how many signal bands we transmit or how large the virtual signal bandwidth is.

In addition, the precision of time delay estimation depends on the width of the secondary main lobe, which is related to the virtual signal bandwidth. As shown in Fig. 4(a), the first secondary zero point of the correlation function of the two edge signal bands (i.e., $100 \mathrm{MHz}$ virtual signal bandwidth, and $20 \mathrm{MHz}$ occupied bandwidth) is about $0.05 T_{s}$, corresponding to $1.5 \mathrm{~m}$ in distance which is much smaller than the one from a single band signal (i.e., $10 \mathrm{MHz}$ (virtual) signal bandwidth). Hence, a multiband signal sparsely occupying the available bandwidth can very much improve the ranging precision.

If the central carrier spacing $\Delta f_{c}$ is not equal across all bands, we may not obtain a closed-form expression of the correction function. The first secondary zero point of the correlation function is determined by the Gabor bandwidth, instead of the actual carrier spacing $\Delta f_{c}$. As shown in (19), the first zero point varies in different signal patterns. In addition, given a non-equal central carrier spacing across different bands, (20) will be no longer valid. Thus, the secondary correlation function will not be periodic, thus it will be different from the one shown in Fig. 4.

\section{Multipath resistance}

In a multipath-free or a low multipath environment and a relatively high SNR, we can simply use multiple signal bands which are sparsely placed in the available bandwidth for ranging and positioning. However, considering 'close-in' multipath, since the product of $C_{r s}(\tilde{\tau})$ still relies on the primary main lobe and contains larger side lobes as shown in Fig. 4(a), the robustness of time delay estimation will not be significantly improved by using multiband signal sparsely occupying the available signal bandwidth. When a relative delay of the reflections is less than the width of the primary main lobe, more sub-bands should be used for ranging and positioning. So that the correlation function has relatively small amplitude of the secondary main lobe (see Fig. 4(b,c)). Reducing the central carrier spacing enlarges the period of the secondary main lobe $T_{0}^{\prime}$. For instance, when ten consecutive 10 MHz sub-bands (i.e., from the band $\{0\}$ to the band $\{9\}$ in Fig. 3) with a central carrier spacing of $10 \mathrm{MHz}$ are used, there is only one secondary main lobe inside the primary main lobe (see Fig. 4 (c)). Therefore, ranging based on these ten bands is more robust to 'close-in' multipath compared with that based on a two sparse edge bands, because less secondary main lobes appear in the primary main lobe. 


\section{TIME DELAY ESTIMATION BASED ON SPARSE MULTIBAND SIGNALS}

As shown in the previous section, the precision of time delay estimation is determined by the virtual signal bandwidth. However, the resistance to multipath relies on the central carrier spacing $\Delta f_{c}$. In order to mitigate the impact of multipath on matched filter based time delay estimation, we need to transmit multiple signal bands which are densely placed in the available spectrum, so that there are only one secondary main lobe and small side lobes inside the primary main lobe (e.g., in Fig. 4(c)).

Considering spectrum efficiency and depending on the actual multipath channel, it may not be necessary to occupy an entire wide signal bandwidth. For example, if we use ten consecutive bands which contain five equally spaced pilots in each band, the width of the secondary main lobe is about $0.1 T_{s}$. Given a $10 \mathrm{MHz}$ baseband signal bandwidth, the impact of multipath that is $3 \mathrm{~m}$ away from the LoS path has been already mitigated significantly, when we apply the matched filter method to estimate the propagation delay. This assumption is still likely realistic in an outdoor scenario. In order to even further improve the accuracy of time delay estimation, we can simply transmit two groups of multiband signals that are sparsely located in the spectrum (e.g., shown in Fig. 5 ), which is quoted as a sparse multiband signal in this paper. Then, a tertiary main lobe appears because of the spacing $\Delta F_{c}$. Increasing the spacing $\Delta F_{c}$ also extends the virtual signal bandwidth, and further narrows the main lobe of the cross correlation function. The ranging precision, consequently, can be further improved.

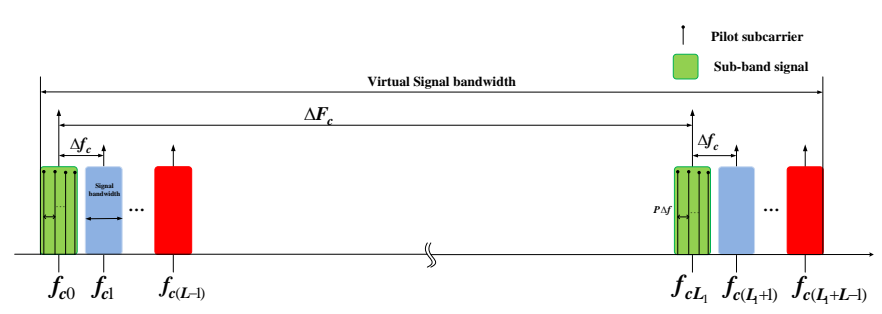

Figure 5: Spectrum of two groups of multiband signals, which are sparsely located, with a spacing of $\Delta F_{c}$. This signal is referred to as a sparse multiband signal.

\section{SAMPLING FREQUENCY OFFSET}

In practice, all transmitters can be synchronized by the same clock source, however, the receiver may run on its own clock. Consequently, a carrier frequency offset and a sampling frequency offset (often referred to as sampling clock offset, SCO) will be introduced in the system. The carrier frequency offset $\hat{\epsilon}$ can be estimated, for example, based on maximum likelihood estimation [16], and can also be easily corrected in time domain by multiplying $\exp (j 2 \pi \hat{\epsilon} n / N)$ on the sampled signal. Although the sampling clock and the carrier frequency stem from the same clock source, we may not retrieve the sampling frequency offset directly from the carrier frequency offset due to Doppler effects on the received signal. In addition, compensation for the impact of the sampling frequency offset requires an extra procedure or hardware components. It can be either corrected on every subcarrier in the frequency domain after the Fourier transform, or by introducing a digital phase-lock loop (DPLL) to correct the sampling frequency offset $[17,18]$. In this section, we only consider a relatively simple receiver that only compensates the carrier frequency offset directly on the sampled signal, but the sampling frequency offset still remains.

Hence, a sampling frequency offset $\eta$ should be taken into account for time delay estimation. The sampling interval $T_{s}^{\prime}$ at the receiver [17] is defined by

$$
T_{s}^{\prime}=(1+\eta) T_{s}
$$

The received signal with a sampling frequency offset is given by

$$
r_{l}[n]=\frac{1}{\sqrt{M}} \sum_{i=-M / 2}^{M / 2-1} c_{i} \exp \left(j 2 \pi \frac{i \operatorname{Pn}(1+\eta)}{N}\right) \exp \left(-j 2 \pi\left(i \Delta f+f_{c}+l \Delta f_{c}\right) \tau T_{s}\right),
$$

and the reference signal is locally generated by (16).

\section{Single band OFDM signal}

We first analyze the impact of the sampling frequency offset on time delay estimation based on a single band OFDM signal. Considering, as before, an OFDM symbol with a BPSK modulation on every pilot subcarrier for time delay estimation, the 
correlation function affected by the sampling frequency offset is derived by

$$
\begin{aligned}
R_{r s, l}(\tilde{\tau}) & =\frac{1}{N} \sum_{n=0}^{N-1} r_{l}[n] s_{l}^{*}[n, \tilde{\tau}] \\
& =\frac{\exp \left(-j 2 \pi\left(f_{c} \tau+l \Delta f_{c}(\tau-\tilde{\tau})\right) T_{S}\right)}{N M} \sum_{i=-M / 2}^{M / 2-1} \sum_{k=-M / 2}^{M / 2-1} \exp \left(-j 2 \pi \frac{i \tau-k \tilde{\tau}}{N} P\right) \sum_{n=0}^{N-1} \exp \left(j 2 \pi \frac{i n-k n+i \eta n}{N} P\right)
\end{aligned}
$$

When the sampling frequency offset $\eta$ is less than certain value (e.g., $200 \mathrm{ppm}$, considering the system used for simulations in section VII), the inter-carrier interference due to the sampling frequency offset can be neglected in (22). In such a case, the correlation function can be further approximated by

$$
\begin{aligned}
R_{r s, l}(\tilde{\tau}) & =\frac{\exp \left(-j 2 \pi\left(f_{c} \tau+l \Delta f_{c}(\tau-\tilde{\tau})\right) T_{s}\right)}{N M} \sum_{i=-M / 2}^{M / 2-1} \exp \left(-j 2 \pi \frac{i(\tau-\tilde{\tau})}{N} P\right) \sum_{n=0}^{N-1} \exp \left(-j 2 \pi \frac{i \eta n}{N} P\right) \\
& \approx \frac{\exp \left(-j 2 \pi\left(f_{c} \tau+l \Delta f_{c}(\tau-\tilde{\tau})\right) T_{s}\right)}{M} \sum_{i=-M / 2}^{M / 2-1} \exp \left(-j 2 \pi \frac{i P}{N}\left(\tau-\tilde{\tau}+\frac{\eta(N-1)}{2}\right)\right) .
\end{aligned}
$$

Hence, due to the sampling frequency offset, the propagation delay is derived by

$$
\hat{\tau}=\underset{\tilde{\tau}}{\arg \min }\left|R_{r s, l}(\tilde{\tau})\right|=\tau+\frac{\eta(N-1)}{2},
$$

which is normalized by the sampling interval $T_{s}$. The error of time delay estimation due to the sampling frequency offset is determined by $\eta$, and also the observation length. Here, only one OFDM symbol (i.e., $N$ samples) is used for time delay estimation and ranging. The more symbols we use, the larger the bias will be.

The sampling frequency offset may not be constant over a very long observation period in practice, but it can be still approximated by a constant value in a certain time interval (e.g., few minutes) [19]. Since all transmitters are assumed to be synchronized by the same clock source, only one sampling frequency offset $\eta$ needs to be estimated at the receiver. In addition, since the receiver may not know the exact transmission time of the signal from the transmitters, the receiver can generate the reference signal at an arbitrary time epoch. Thus, an initial clock offset should be also taken into account.

For the purpose of positioning, we can either estimate the clock error being the sum of the initial clock offset and the bias introduced by the sampling frequency offset (24) in the positioning model as in GNSS, or apply the time-difference-of-arrival (TDoA) approach to eliminate the impact of the clock error.

\section{Multiband OFDM signal}

Now considering a case of using multiple signal bands for time delay estimation and ranging. If all signal bands are transmitted and received simultaneously on a single transmitter-receiver link, we can simply take a snap shot of the received signals for time delay estimation. Here, we simply assume that besides the initial clock offset and the sampling frequency offset as we discussed in the previous subsection, no extra timing error is introduced in the received signal. The received signals and the locally generated signals are given by (21) and (16), respectively. Therefore, the correlation affected by the sampling clock offset can be further derived as follows

$$
\begin{aligned}
R_{r s}(\tilde{\tau})= & \frac{1}{L} \sum_{l=0}^{L-1} R_{r s, l}(\tilde{\tau}) \approx \exp \left(-j 2 \pi f_{c} \tau T_{s}\right) \frac{1}{L} \sum_{l=0}^{L-1} \exp \left(-j 2 \pi l \Delta f_{c}(\tau-\tilde{\tau}) T_{s}\right) \frac{1}{M} \sum_{i=-M / 2}^{M / 2-1} \exp \left(-j 2 \pi \frac{i P}{N}\left(\tau-\tilde{\tau}+\frac{\eta(N-1)}{2}\right)\right) \\
= & \exp \left(-j 2 \pi f_{c} \tau T_{s}\right) \exp \left(-j 2 \pi \Delta f_{c}(\tau-\tilde{\tau}) T_{s} \frac{L-1}{2}\right) \frac{1}{L} \frac{\sin \left(2 \pi \Delta f_{c}(\tau-\tilde{\tau}) T_{s} \frac{L}{2}\right)}{\sin \left(2 \pi \Delta f_{c}(\tau-\tilde{\tau}) T_{s} \frac{1}{2}\right)} \\
& \exp \left(-j 2 \pi \frac{P(M-1)}{2 N}\left(\tau-\tilde{\tau}+\frac{\eta(N-1)}{2}\right)\right) \exp \left(j 2 \pi \frac{M P}{2 N}\left(\tau-\tilde{\tau}+\frac{\eta(N-1)}{2}\right)\right) \frac{1}{M} \frac{\sin \left(2 \pi \frac{P M}{2 N}\left(\tau-\tilde{\tau}+\frac{\eta(N-1)}{2}\right)\right)}{\sin \left(2 \pi \frac{P}{2 N}\left(\tau-\tilde{\tau}+\frac{\eta(N-1)}{2}\right)\right)}
\end{aligned}
$$


Consequently, the amplitude of the correlation function is given by

$$
C_{r s}(\tilde{\tau})=\left|R_{r s}(\tilde{\tau})\right|=|\underbrace{\frac{1}{L} \frac{\sin \left(2 \pi \Delta f_{c}(\tau-\tilde{\tau}) T_{s} \frac{L}{2}\right)}{\sin \left(2 \pi \Delta f_{c}(\tau-\tilde{\tau}) T_{s} \frac{1}{2}\right)}}_{C_{r s, 2}(\tilde{\tau})} \underbrace{\frac{1}{M} \frac{\sin \left(2 \pi \frac{P M}{2 N}\left(\tau-\tilde{\tau}+\frac{\eta(N-1)}{2}\right)\right)}{\sin \left(2 \pi \frac{P}{2 N}\left(\tau-\tilde{\tau}+\frac{\eta(N-1)}{2}\right)\right)}}_{C_{r s, 1}(\tilde{\tau})}| .
$$

Similar to (18), the correlation function derived in (26) contains a primary main lobe and the secondary main lobe. Due to the sampling frequency offset, the primary correlation function $C_{r s, 1}(\tilde{\tau})$ is shifted from the primary correlation function in (18). However, the actual carrier phase is not affected by the sampling frequency offset, thus, the secondary correlation function $C_{r s, 2}(\tilde{\tau})$ still remains the same as in (18). In addition, the envelope of the secondary main lobe in the product of $C_{r s}(\tilde{\tau})$ is determined by the primary main lobe. For example in Fig. 6, when ten consecutive bands are used, the peak in the correlation is attenuated due to the sampling frequency offset, but its actual location is not moved. Thus, aggregating multiple narrowband signals can mitigate the bias of time delay estimation, when the signals from multiple bands are acquired simultaneously.

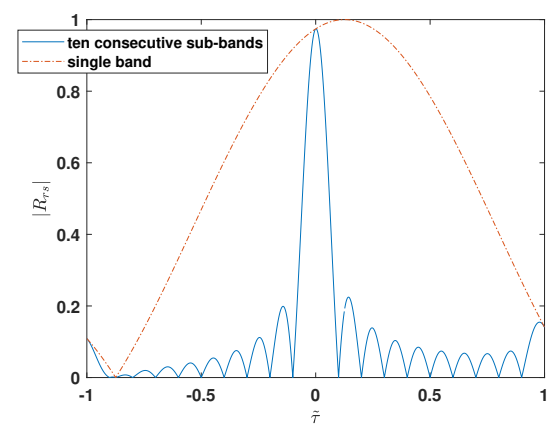

Figure 6: Cross correlation based on a single band OFDM signal and ten consecutive signal bands, which are received simultaneously, in the presence of a sampling frequency offset.

However, a timing synchronization should be independently carried out for each band, if the signals from different bands are received sequentially. When the variation of timing synchronization across different bands can be restricted to \pm 1 sample interval, the impact of the sampling frequency offset can be still mitigated, since the central carrier phase from different bands are not affected by the sampling frequency offset.

\section{SIMULATION}

First, we evaluate the impact of a sampling frequency offset in a simple LoS propagation channel. As we discussed in section VI, the sampling frequency offset will only perturb the subcarrier phase, the central carrier phase is not affected and remains 'clean'. Therefore, using multiple signals from different bands can mitigate the time delay estimation error introduced by the sampling frequency offset. For comparison, a single band OFDM (i.e., band: $\{0\}$ in Fig. 3, $10 \mathrm{MHz}$ bandwidth), two consecutive bands (i.e., band: $\{0,1\}, 20 \mathrm{MHz}$ bandwidth), five consecutive bands (i.e., band: $\{0-4\}, 50 \mathrm{MHz}$ bandwidth), and ten consecutive sub-bands (i.e., band: $\{0-9\}, 100 \mathrm{MHz}$ bandwidth) are considered as ranging signals for time delay estimation, respectively. Each band has $10 \mathrm{MHz}$ signal bandwidth, and simply let all subcarriers (i.e., $N=64$ ) be pilot subcarriers for time delay estimation. In addition, the central carrier spacing $\Delta f_{c}$ in (16) is also $10 \mathrm{MHz}$. In the simulation, we also assume that the signals from different bands can be received either simultaneously, or sequentially based on the time stamping.

Then, Fig. 7 shows the ranging error due to a sampling frequency offset. For example, using a single OFDM symbol from a single band signal for time delay estimation, a 100 ppm sampling frequency offset can cause about 0.1 m ranging error. Generally, the ranging error is in line with the error derived in (24). When we aggregate a multiple sub-band signal, the larger the virtual signal bandwidth is, the sharper the secondary main lobe becomes. Thus, the maximum of the correlation function becomes less sensitive to the sampling frequency offset as we discussed in section VI. However, it is worth to mention that a very sparse multiband signal (e.g., only the band: $\{0,9\}$ shown in Fig. 3) will result in large side lobes (see Fig. 4(a)), because of the small secondary period of the correlation function. Even though it is insensitive to a sampling clock offset, time delay estimation can, 


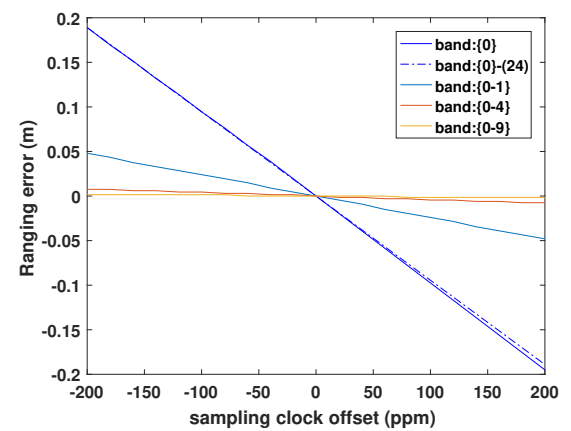

Figure 7: Ranging error because of a sampling frequency offset in an ideal channel (i.e., no noise and multipath), when the ranging signal consists of a single band OFDM signal (e.g. band: $\{0\}$ ), two consecutive bands (e.g. band: $\{0-1\}$ ), five consecutive bands (e.g. band:\{0-4\}), and ten consecutive bands (e.g. band:\{0-9\}), respectively. Each band has a $10 \mathrm{MHz}$ signal bandwidth, and all subcarrier (i.e., $N=64$ ) are used for time delay estimation and ranging. The central carrier spacing $\Delta f_{c}$ is $10 \mathrm{MHz}$. For comparison, the ranging error due to the sampling clock offset in a single band OFDM signal derived in (24) is shown as a dashed line, which is very close the empirical ranging error shown as a solid line.

then, be easily affected by the noise and multipath.

To evaluate the performance of time delay estimation in different SNRs, the signal power in different bands is assumed to be the same, and the sampling frequency offset $\eta$ is fixed to $100 \mathrm{ppm}$. We begin with a simple single path channel. Since time delay estimation is biased, Fig. 8 shows the root mean square error (RMSE) of the estimate, instead of the standard deviation. Due to the bias from the sampling frequency offset, ranging based on a single $10 \mathrm{MHz}$ OFDM signal contains a mean of 0.1 $\mathrm{m}$ as derived in (24), and can approximately achieve a meter-level accuracy. Aggregating ten consecutive bands can emulate a $100 \mathrm{MHz}$ (virtual) signal bandwidth and achieve a centimeter level ranging accuracy. Furthermore, as shown in Fig. 5, we can transmit two groups of multiband signals, which are sparsely placed in the available spectrum to further increase the virtual signal bandwidth. Therefore, using the signal occupying the band $\{0-9\}$ and the band $\{40-49\}$ can eventually lead to a sub-centimeter level ranging accuracy (shown in black line in Fig. 8), which is also more accurate than using the same effective bandwidth (i.e., $200 \mathrm{MHz}$ ) with twenty consecutive bands (shown in blue line in Fig. 8).

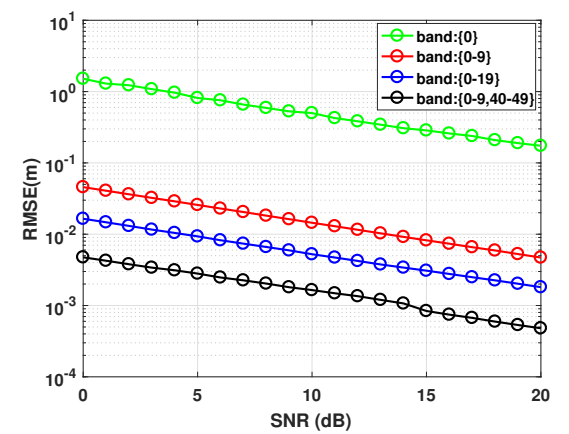

Figure 8: Root mean square error of ranging based on a single band OFDM signal (e.g. band: $\{0\}$ ), ten consecutive bands (e.g. band: $\{0-9\}$ ), twenty consecutive bands (e.g. band:\{0-19\}), and two groups of multiband signals shown in Fig. 5 (e.g. band: $\left.\{0-9,40-49\}, \Delta F_{c}=400 \mathrm{MHz}\right)$, respectively. The RMSE is computed based on 1000 independent simulations. In addition, the sampling frequency offset is fixed to $100 \mathrm{ppm}$.

In order to further evaluate the performance of time delay estimation in a multipath condition, a multipath channel is generated as that rays arrive in clusters [20,21]. This paper only considers a direct-LoS multipath channel, thus, the LoS path is always fixed as the first path in the channel impulse response. As indicated in [20], the arrival time of the clusters and the paths satisfy Poisson distributions. In addition, the cluster rate $\Lambda$ is 0.033 , and the ray arrival rate $\lambda$ is 0.5 , the cluster power-decay time constant $\Gamma$ is 20.4, and the ray power-decay time constant $\gamma$ is 0.2 . In addition, no close-in multipath (i.e., less than $3 \mathrm{~m}$ away from the LoS path) is generated in the channel impulse response. Considering a $10 \mathrm{MHz}$ sub-band signal, the sampling interval $T_{s}$ is $10^{-7} \mathrm{~s}$, as an example, Fig. 9(a) shows one of the channel impulse responses for the simulation. An independent channel impulse response is generated in each simulation. But the locally generated template used for the matched filter contains only a single path, see 
(16).

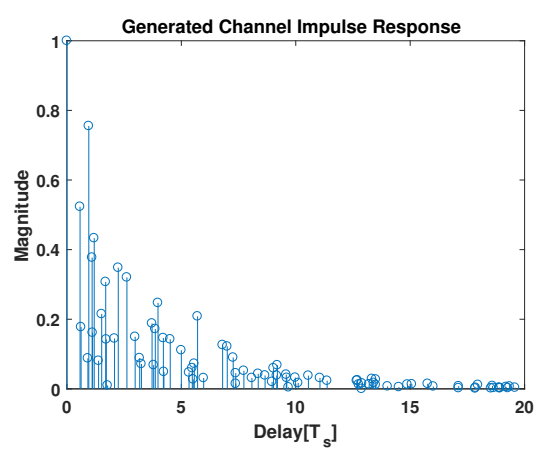

(a)

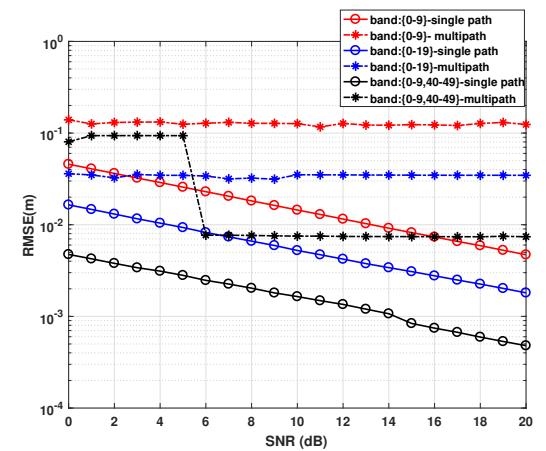

(b)

Figure 9: (a) An example of a generated channel impulse response, the LoS path has magnitude 1 at delay $0 T_{s}$. All reflections are assumed to be at least $3 \mathrm{~m}$ away from the LoS path (i.e., the relative delay of reflections is always larger than $0.1 T_{s}$ ). (b) Root mean square error of ranging in a simple single path channel which are identical to Fig. 8, and multipath channel based ten consecutive bands (e.g. band: $\{0-9\}$ ), twenty consecutive bands (e.g. band:\{0-19\}), and two groups of multiband signals shown in Fig. 5 (e.g. band: $\{0-9,40-49\}, \Delta F_{c}=400 \mathrm{MHz}$ ), respectively. In addition, the RMSE is computed from 1000 simulations and the sampling frequency offset is fixed to $100 \mathrm{ppm}$.

For comparison, Fig. 9(b) shows the RMSE of ranging in both a simple single path channel and a multipath channel. As discussed in section IV, aggregating ten consecutive bands can further improve the resistance of multipath compared with that based on a single band signal. Therefore, time delay estimation based on a multiband signal is still robust in the multipath condition, although the ranging error now is dominated by the residual multipath error. And the sparse-multi multiband signal discussed in section $\mathrm{V}$ can further improve the ranging accuracy up to a centimeter level in multipath environments. With two groups of multiband signals, each group covers $10 \times 10 \mathrm{MHz}$ and is separated by $400 \mathrm{MHz}$, the RMSE is about $0.007 \mathrm{~m}$ in the given multipath channel and $0.002 \mathrm{~m}$ in single path channel, when SNR is $10 \mathrm{~dB}$.

When a sparse multiband signal is used, in a low SNR and multipath, however, the side lobe may become even larger than the actual main lobe. This will cause a false or incorrect detection of the maximum and introduce a bias of about $0.025 T_{s}$ and $0.05 T_{s}$ on time delay estimation. Thus, in Fig. 9(b), when the SNR is $5 \mathrm{~dB}$, the RMSE is about $0.09 \mathrm{~m}$ due to the bias introduced by incidental false or incorrect detection. Based on the multipath model we used in the simulation, the propagation delay is incorrectly determined in 7 out of 1000 simulations namely through a side lobe.

\section{CONCLUSION}

Based on a multi-carrier signal (i.e., OFDM), we investigate the signal characteristics for the purpose of time delay estimation and ranging. Precision, ambiguity and resistance in a multipath channel are taken into account. We suggest that the allocation of ranging pilots (i.e., the signal spectrum) and multiple signal bands to be guided by the following steps:

- In accordance with the required un-ambiguous ranging distance and the ranging scenario (i.e., channel model), determine the minimum number of pilots in a single band signal for time delay estimation.

- Successively or simultaneously transmit multiple signal bands to further mitigate the impact of close-in multipath on time delay estimation. The spacing of the central carriers of the various bands and the number of bands are determined by the maximum tolerable multipath error and the channel.

- Transmit a sparse multiband signal consisting of two groups of multiband signal that are well separated in the available spectrum (e.g., in Fig. 5) to further narrow the main lobe of the cross correlation function. The spacing of these two groups of sub-band signals is determined by the required ranging accuracy and the possible SNR.

Using the proposed sparse multiband signals, simulation results show that a centimetre-level ranging accuracy can be achieved in the presence of dense multipath and a sampling frequency offset. Further research will include the optimization of the multiband signal design with a non-uniform central carrier spacing, and a non-identical power distribution on pilot subcarriers within a single band OFDM signal. 


\section{ACKNOWLEDGEMENT}

This research is supported by the Netherlands Organization for Scientific Research (NWO) through the project 'SuperGPS' under Grant 13970.

\section{A. CROSS CORRELATION OF PILOT SUBCARRIERS}

Based on received signal and the locally generated reference signal (10) with BPSK modulations, the cross-correlation is written by

$$
\begin{aligned}
R_{r s}(\tilde{\tau})= & \frac{1}{N} \sum_{n=0}^{N-1} \frac{1}{\sqrt{M}} \sum_{i=0}^{M-1} \exp \left(j 2 \pi \frac{i P}{N}(n-\tau)\right) \exp \left(-j 2 \pi \frac{M P}{2 N}(n-\tau)\right) \exp \left(-j 2 \pi f_{c} \tau T_{s}\right) \\
& \cdot \frac{1}{\sqrt{M}} \sum_{k=0}^{M-1} \exp \left(-j 2 \pi \frac{k P}{N}(n-\tilde{\tau})\right) \exp \left(j 2 \pi \frac{M P}{2 N}(n-\tilde{\tau})\right) \\
= & \frac{\exp \left(-j 2 \pi f_{c} \tau T_{s}\right)}{N M} \exp \left(j 2 \pi \frac{M P}{2 N}(\tau-\tilde{\tau})\right) \sum_{i=0}^{M-1} \sum_{k=0}^{M-1} \exp \left(-j 2 \pi \frac{(i \tau-k \tilde{\tau}) P}{N}\right) \sum_{n=0}^{N-1} \exp \left(j 2 \pi \frac{i-k}{N} P n\right),
\end{aligned}
$$

where $P$ stands for the pilot spacing, $M$ denotes the number of pilots out of $N$ subcarriers in the OFDM signal. since

$$
\sum_{n=0}^{N-1} \exp \left(j 2 \pi \frac{i-k}{N} n\right)=\frac{\sin \left(2 \pi \frac{i-k}{2}\right)}{\sin \left(2 \pi \frac{i-k}{2 N}\right)} \exp \left(j 2 \pi \frac{i-k}{N} \frac{N-1}{2}\right), \text { and } \frac{\sin \left(2 \pi \frac{i-k}{2}\right)}{\sin \left(2 \pi \frac{i-k}{2 N}\right)}=\left\{\begin{array}{ll}
N, & i=k \\
0, & i \neq k
\end{array},\right.
$$

we can further simplify (27) as follows

$$
\begin{aligned}
R_{r s}(\tilde{\tau}) & =\frac{\exp \left(-j 2 \pi f_{c} \tau T_{s}\right)}{M} \exp \left(j 2 \pi \frac{M P}{2 N}(\tau-\tilde{\tau})\right) \sum_{i=0}^{M-1} \exp \left(-j 2 \pi \frac{i(\tau-\tilde{\tau})}{N} P\right) \\
& =\frac{\exp \left(-j 2 \pi f_{c} \tau T_{s}\right)}{M} \exp \left(j 2 \pi \frac{M P}{2 N}(\tau-\tilde{\tau})\right) \exp \left(-j 2 \pi \frac{P}{N} \frac{M-1}{2}(\tau-\tilde{\tau})\right) \frac{\sin \left(2 \pi \frac{P}{N} \frac{M}{2}(\tau-\tilde{\tau})\right)}{\sin \left(2 \pi \frac{P}{N} \frac{1}{2}(\tau-\tilde{\tau})\right)} .
\end{aligned}
$$

\section{B. CROSS CORRELATION OF MULTIBAND OFDM SIGNALS}

Assuming each band has the same modulation given by (10), so the received signal from the $l$-th band is written by

$$
\begin{aligned}
r_{l}[n] & =\frac{\alpha_{l}}{\sqrt{M}} \sum_{i=M / 2}^{M / 2-1} \exp \left(j 2 \pi \frac{i P}{N} n\right) \exp \left(-j 2 \pi\left(i P \Delta f+l \Delta f_{c}+f_{c}\right) \tau T_{s}\right) \\
& =\frac{\alpha_{l}}{\sqrt{M}} \sum_{i=0}^{M-1} \exp \left(j 2 \pi \frac{i P}{N}(n-\tau)\right) \exp \left(-j 2 \pi \frac{M P}{2 N}(n-\tau)\right) \exp \left(-j 2 \pi\left(l \Delta f_{c}+f_{c}\right) \tau T_{s}\right) .
\end{aligned}
$$

In addition, if the multiband signal only appears in a certain span of the spectrum (e.g., $500 \mathrm{MHz}$ ), the attenuation $\alpha$ is approximately assumed to be the same. Combining the correlation function based on a single band OFDM signal (29), the correlation function based on the multiband signal (17) can be rewritten by

$$
\begin{aligned}
R_{r s}(\tilde{\tau})= & \frac{\exp \left(-j 2 \pi f_{c} \tau T_{s}\right)}{M} \exp \left(j 2 \pi \frac{M P}{2 N}(\tau-\tilde{\tau})\right) \exp \left(-j 2 \pi \frac{P}{N} \frac{M-1}{2}(\tau-\tilde{\tau})\right) \frac{\sin \left(2 \pi \frac{P}{N} \frac{M}{2}(\tau-\tilde{\tau})\right)}{\sin \left(2 \pi \frac{P}{N} \frac{1}{2}(\tau-\tilde{\tau})\right)} \\
& \frac{1}{L} \sum_{l=0}^{L-1} \exp \left(-j 2 \pi l \Delta f_{c}(\tau-\tilde{\tau}) T_{s}\right) \\
= & \frac{\exp \left(-j 2 \pi f_{c} \tau T_{s}\right)}{M} \exp \left(j 2 \pi \frac{M P}{2 N}(\tau-\tilde{\tau})\right) \exp \left(-j 2 \pi \frac{P}{N} \frac{M-1}{2}(\tau-\tilde{\tau})\right) \frac{\sin \left(2 \pi \frac{P}{N} \frac{M}{2}(\tau-\tilde{\tau})\right)}{\sin \left(2 \pi \frac{P}{N} \frac{1}{2}(\tau-\tilde{\tau})\right)} \\
& \frac{1}{L} \exp \left(-j 2 \pi \Delta f_{c}(\tau-\tilde{\tau}) T_{s} \frac{L-1}{2}\right) \frac{\sin \left(2 \pi \Delta f_{c}(\tau-\tilde{\tau}) T_{s} \frac{L}{2}\right)}{\sin \left(2 \pi \Delta f_{c}(\tau-\tilde{\tau}) T_{s} \frac{1}{2}\right)} .
\end{aligned}
$$




\section{BIBLIOGRAPHY}

[1] P. Teunissen and O. Montenbruck, "Signals and modulation," in Springer handbook of global navigation satellite systems, ch. 4, Springer, 2017.

[2] J.-Y. Lee and R. A. Scholtz, "Ranging in a dense multipath environment using an UWB radio link," IEEE Journal on Selected Areas in Communications, vol. 20, no. 9, pp. 1677-1683, 2002.

[3] H. Xu, C.-C. Chong, I. Guvenc, F. Watanabe, and L. Yang, "High-resolution TOA estimation with multi-band OFDM UWB signals," in 2008 IEEE International Conference on Communications, pp. 4191-4196, IEEE, 2008.

[4] F. Wolf, J.-B. Doré, X. Popon, S. de Rivaz, F. Dehmas, and J.-P. Cances, "Coherent multi-channel ranging for narrowband LPWAN: Simulation and experimentation results," in 2018 15th Workshop on Positioning, Navigation and Communications (WPNC), pp. 1-6, IEEE, 2018.

[5] D. Vasisht, S. Kumar, and D. Katabi, "Decimeter-level localization with a single WiFi access point," in 13th Symposium on Networked Systems Design and Implementation, pp. 165-178, 2016.

[6] C. Yang and A. Soloviev, "Pinch the correlation function: A method to improve delay estimation in multipath," in Proceeding of the 2017 International Technical Meeting, ION ITM 2017, pp. 347-364, ION, 2017.

[7] S. M. Kay, Fundamentals of statistical signal processing. Prentice Hall PTR, 1993.

[8] L. Giugno and M. Luise, "Optimum pulse shaping for delay estimation in satellite positioning.," in EUSIPCO, pp. 1-6, 2005.

[9] J. W. Betz, "Binary offset carrier modulations for radionavigation," Navigation, vol. 48, no. 4, pp. 227-246, 2001.

[10] J. A. del Peral-Rosado, J. A. López-Salcedo, F. Zanier, and G. Seco-Granados, "Position accuracy of joint time-delay and channel estimators in LTE networks," IEEE Access, vol. 6, pp. 25185-25199, 2018.

[11] G. Kalverkamp, B. Schaffer, and E. Biebl, "OFDM-based ranging approach for vehicular safety applications," in 2013 IEEE 78th Vehicular Technology Conference (VTC Fall), pp. 1-5, IEEE, 2013.

[12] Y. G. Li and G. L. Stuber, "Basic concept," in Orthogonal frequency division multiplexing for wireless communications, ch. 2, Springer Science \& Business Media, 2006.

[13] R. K. Martin, C. Yan, and H. H. Fan, "Bounds on distributed TDOA-based localization of OFDM sources," in Acoustics, Speech and Signal Processing, 2009. ICASSP 2009. IEEE International Conference on, pp. 2289-2292, IEEE, 2009.

[14] M. Driusso, M. Comisso, F. Babich, and C. Marshall, "Performance analysis of time of arrival estimation on OFDM signals," IEEE Signal Processing Letters, vol. 22, no. 7, pp. 983-987, 2015.

[15] P. Misra and P. Enge, "Global Positioning System: signals, measurements and performance second edition," Massachusetts: Ganga-Jamuna Press, 2006.

[16] J.-J. Van de Beek, M. Sandell, and P. O. Borjesson, "ML estimation of time and frequency offset in OFDM systems," IEEE transactions on signal processing, vol. 45, no. 7, pp. 1800-1805, 1997.

[17] Y. G. Li and G. L. Stuber, "Synchronization," in Orthogonal frequency division multiplexing for wireless communications, ch. 4, Springer Science \& Business Media, 2006.

[18] T. Pollet, P. Spruyt, and M. Moeneclaey, “The BER performance of OFDM systems using non-synchronized sampling," in 1994 IEEE GLOBECOM. Communications: The Global Bridge, pp. 253-257, IEEE, 1994.

[19] J. Khalife and Z. Kassas, "Evaluation of relative clock stability in cellular networks," in Proceedings of ION GNSS Conference, pp. 2554-2559, 2017.

[20] A. A. Saleh and R. Valenzuela, "A statistical model for indoor multipath propagation," IEEE Journal on selected areas in communications, vol. 5, no. 2, pp. 128-137, 1987.

[21] H. Hashemi, "Impulse response modeling of indoor radio propagation channels," IEEE journal on selected areas in communications, vol. 11, no. 7, pp. 967-978, 1993. 